BMJ Open

Diabetes

Research

\& Care

\title{
Secreted factors from dental pulp stem cells improve glucose intolerance in streptozotocin-induced diabetic mice by increasing pancreatic $\beta$-cell function
}

\author{
Takako Izumoto-Akita, ${ }^{1,2}$ Shin Tsunekawa, ${ }^{1}$ Akihito Yamamoto, ${ }^{2}$ Eita Uenishi, ${ }^{1}$ \\ Kota Ishikawa, ${ }^{1}$ Hidetada Ogata, ${ }^{1}$ Atsushi lida, ${ }^{1}$ Makoto Ikeniwa, ${ }^{1}$ \\ Kaori Hosokawa, ${ }^{1}$ Yasuhiro Niwa, ${ }^{1}$ Ryuya Maekawa, ${ }^{1}$ Yuichiro Yamauchi, ${ }^{1}$ \\ Yusuke Seino, ${ }^{3}$ Yoji Hamada, ${ }^{3}$ Hideharu Hibi, ${ }^{2}$ Hiroshi Arima, ${ }^{1}$ Minoru Ueda, ${ }^{2}$ \\ Yutaka Oiso $^{1}$
}

To cite: Izumoto-Akita T, Tsunekawa S, Yamamoto A, et al. Secreted factors from dental pulp stem cells improve glucose intolerance in streptozotocin-induced diabetic mice by increasing pancreatic $\beta$-cell function. BMJ Open Diabetes Research and Care 2015;3:e000128. doi:10.1136/bmjdrc-2015000128

- Additional material is available. To view please visit the journal (http://dx.doi.org/ 10.1136/bmjdrc-2015000128)

Received 25 June 2015 Revised 25 August 2015 Accepted 23 September 2015

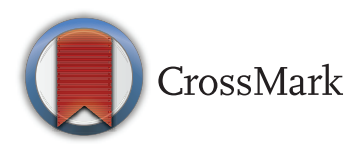

For numbered affiliations see end of article.

Correspondence to Dr Shin Tsunekawa; tsune87@med.nagoya-u.ac.jp

\section{ABSTRACT}

Objective: Many studies have reported that stem cell transplantation promotes propagation and protection of pancreatic $\beta$-cells in streptozotocin (STZ)-induced diabetic mice without the differentiation of transplanted cells into pancreatic $\beta$-cells, suggesting that the improvement is due to a paracrine effect of the transplanted cells. We investigated the effects of factors secreted by dental pulp stem cells from human exfoliated deciduous teeth (SHED) on $\beta$-cell function and survival.

Research design and methods: Conditioned medium from SHED (SHED-CM) was collected $48 \mathrm{~h}$ after culturing in serum-free Dulbecco's modified Eagle's medium (DMEM). The insulin levels in SHED$\mathrm{CM}$ and serum-free conditioned media from human bone marrow-derived mesenchymal stem cells (BM$\mathrm{CM}$ ) were undetectable. STZ-induced diabetic male C57B/6J mice were injected with DMEM as a control, SHED-CM, exendin-4 (Ex-4), or BM-CM for 14 days. Mouse pancreatic $\beta$-cell line MIN6 cells were incubated with different concentrations of STZ with SHED-CM, DMEM, Ex-4, or BM-CM for $6 \mathrm{~h}$.

Results: Administration of $1 \mathrm{~mL}$ of SHED-CM twice a day improved glucose intolerance in STZ-induced diabetic mice and the effect continued for 20 days after the end of treatment. SHED-CM treatment increased pancreatic insulin content and $\beta$-cell mass through proliferation and an intraperitoneal glucose tolerance test revealed enhanced insulin secretion. Incubation of MIN6 cells (a mouse pancreatic $\beta$-cell line) with SHEDCM enhanced insulin secretion in a glucose concentration-dependent manner and reduced STZinduced cell death, indicating that the amelioration of hyperglycemia was caused by the direct effects of SHED-CM on $\beta$-cell function and survival. These effects were more pronounced than with the use of Ex-4, a conventional incretin-based drug, and BM-CM, which is a medium derived from other stem cells.

Conclusions: These findings suggest that SHED-CM provides direct protection and encourages the propagation of $\beta$-cells, and has potential as a novel strategy for treatment of diabetes.

\section{Key messages}

- Secreted factors from stem cells protect $\beta$-cell.

- Secreted factors from stem cells increase insulin secretion.

- Secreted factors from stem cells might be useful as new diabetic treatment.

\section{INTRODUCTION}

Diabetes mellitus is a metabolic disease mainly characterized by chronic hyperglycemia, which is induced either as a result of insulin resistance or impairment of insulin secretion. Since $70-75 \%$ of obese patients do not develop diabetes due to the effect of compensatory insulin secretion, ${ }^{1}$ maintenance of functional pancreatic $\beta$-cells is considered to be the ultimate outcome of diabetes treatment.

Existing antidiabetes drugs are insufficient to suppress progressive damage to pancreatic $\beta$-cells; thus, patients must eventually be switched to insulin therapy. ${ }^{2}$ Therefore, islet transplantation and regenerative medicine continue to receive wide attention as an emerging treatment option for diabetes. ${ }^{3}$ Meanwhile, previous in vivo studies have reported that transplantation of stem cells, including embryonic or mesenchymal stem cells (MSCs), improved hyperglycemia in rodent models of streptozotocin (STZ)-induced diabetes. ${ }^{4}$ Since transplanted stem cells do not differentiate into pancreatic $\beta$-cells, the direct cause of glycemic control is considered to be due to paracrine effects that promote the propagation and protection of pancreatic $\beta$-cells. Similarly, improvements in spinal cord injuries and hypoxic-ischemic brain injuries due to stem cell 
transplantation are reportedly attributable to paracrine effects, in which cytokine diffusion from stem cells plays a vital role. ${ }^{5} 6$ Therefore, the use of secreted factors, which can be collected as a serum-free conditioned medium (CM) of stem cells, without the need for cell transplantation and immunosuppressive agents, has recently become a target of scientific research. ${ }^{78}$

Dental pulp stem cells from human exfoliated deciduous teeth (SHED) have received considerable attention because of the advantages of a less invasive collection method and the applicability to autologous treatment. ${ }^{9}$ SHED are thought to originate from the cranial neural crest, which expresses markers for both embryonic and MSC, and reside within the perivascular niche of the dental pulp. ${ }^{9}{ }^{10}$ SHED also express many genes encoding extracellular and cell surface proteins at levels that are at least twofold higher than those in human bone marrow-derived MSCs (BM-CM). ${ }^{11}{ }^{12}$ Moreover, transplantation of dental pulp stem cells was reported to improve diabetic control in STZ-induced diabetic mice, but the exact underlying mechanism remains unclear. ${ }^{13}$

In this study, we investigated the effect of SHED-CM injections on $\beta$-cell function and survival in STZ-induced diabetic mice. Furthermore, we compared the effects of SHED-CM treatment and treatment with exendin-4 (Ex-4), a conventional incretin-based drug, and human BM-CM, which is a medium derived from other stem cells.

\section{MATERIALS AND METHODS}

\section{Preparation of stem cell-CIM}

Exfoliated deciduous teeth from 6 to 12-year-olds were collected and stored for clinical purposes at Nagoya University Hospital. SHED were collected and cultured as previously described. ${ }^{9}$ BM from 20 to 22-year-olds between passages 10 and 14 was obtained from Lonza and the Health Science Research Resources Bank. Stem cell-CM was prepared as previously described. ${ }^{12}$ Briefly, cells were cultured to $80 \%$ confluence, rinsed three times with phosphate-buffered saline (PBS), and then cultured in serum-free Dulbecco's modified Eagle's medium (DMEM) for $48 \mathrm{~h}$. CM was collected and centrifuged twice for $5 \mathrm{~min}$ at $22140 \times \mathrm{g}$ at $4^{\circ} \mathrm{C}$. The supernatant was collected and used as CM.

\section{In vivo studies}

C57Bl/6J mice (Chubu Kagaku Shizai Co, Ltd, Aichi, Japan) used as test subjects were housed according to a protocol approved by the Nagoya University Institutional Animal Care and Use Committee. To induce pancreatic damage causing diabetes, male mice aged 10 weeks were intraperitoneally injected daily for 5 days with $50 \mathrm{mg} / \mathrm{kg}$ body weight (BW) of STZ (Sigma-Aldrich, St. Louis, Missouri, USA) dissolved in saline (multiple low-dose STZ-induced diabetic mice: MLDS mice). The 20 mice were divided into four treatment groups of five mice each as follows: control (DMEM; $1 \mathrm{~mL}$ injection two times per day); SHED-CM (1 mL injection two times per day); Ex-4 (24 nmol $/ \mathrm{kg}$ BW injection two times per day); and BM-CM (1 mL injection two times per day). Doses were injected intravenously for the first 5-day period, which coincided with the STZ injection period, and then injected intraperitoneally for the subsequent 9 days. These experiments were conducted three times with three different sets of mice. In order to show that the effects were not due to an anti-STZ effect of the treatment, $150 \mathrm{mg} / \mathrm{kg} \mathrm{BW}$ of STZ was injected intraperitoneally into male $\mathrm{C} 57 \mathrm{Bl} / 6 \mathrm{~J}$ mice aged 10 weeks only once (high-dose STZ-induced diabetic mice: HD mice). Three days after this single STZ injection, HD mice with random plasma glucose levels of $\geq 20 \mathrm{mmol} / \mathrm{L}$ were divided into three treatment groups: control (DMEM), SHED-CM, and Ex-4 dose, as described previously.

\section{Animal monitoring}

Random plasma glucose levels and BWs were measured in ad libitum fed mice every day at 09:00. Plasma glucose levels were measured using the Antsense III glucose analyzer (Horiba, Ltd, Kyoto, Japan).

\section{Glucose tolerance tests}

After fasting for $16 \mathrm{~h}, 2.0 \mathrm{~g} / \mathrm{kg}$ BW of D-glucose was injected intraperitoneally into the treated mice. Plasma glucose levels were then measured using the Antsense III glucose analyzer. Plasma insulin levels were measured using a mouse insulin ELISA kit (Morinaga Institute of Biological Science, Tokyo, Japan).

\section{Measurements of total pancreas insulin content}

The pancreas was isolated from each mouse as previously described. ${ }^{14}$ Total pancreas insulin content was measured according to a standard acid-ethanol extraction protocol ${ }^{15}$ using the H.T.R.F insulin assay (Cisbio Bioassays, Codolet, France).

\section{Histological analysis}

Each isolated pancreas was fixed in $4 \%$ paraformaldehyde, embedded in paraffin, and sectioned. Frozen sections were prepared as previously described. ${ }^{15}$ Briefly, the sections were incubated at $4^{\circ} \mathrm{C}$ overnight with guinea pig anti-insulin antibody and either rabbit antiglucagon antibody or rabbit anti-Ki67 antibody (dilution, 1:500; Abcam, Tokyo, Japan). Afterward, the sections were washed with PBS, then further incubated for $1 \mathrm{~h}$ in a mixture of rhodamine-conjugated antiguinea pig IgG antibody, antirabbit IgG antibody, and DAPI (4',6-diamidino-2-phenylindole) fluorescent stain solution (dilution, 1:2000; Dojindo Molecular Technologies, Inc, Tokyo, Japan).

For morphological analysis, five sections per pancreas were randomly selected at $150 \mu \mathrm{m}$ intervals. The sections were analyzed for cell count and cell area using a BZ-9000 fluorescent microscope system (Keyence, Osaka, Japan). 


\section{Cell culture and viability}

MIN6 cells (a mouse pancreatic $\beta$-cell line) were obtained as a gift from Junichi Miyazaki (Osaka University, Osaka, Japan), ${ }^{16}$ cultured to $80 \%$ confluence, and then incubated for $6 \mathrm{~h}$ with different concentrations of STZ $(0,1$, or $5 \mathrm{mmol} / \mathrm{L})$ with SHED-CM, DMEM, Ex-4 $(10 \mathrm{nmol} / \mathrm{L})$, or BM-CM.
In vitro immunofluorescent staining was performed following almost the same procedure as that for the in vivo study. The MIN6 cells were defined by shape and nuclear diameter, in which there were a greater number of early stage necrotic cells than were observed in normal MIN6 cells $(7.16 \pm 0.59 \mu \mathrm{m})$, using the BZ 9000 fluorescent microscope system. ${ }^{17}{ }^{18}$ In addition, cell death was


Figure 1 Change in random blood glucose level, body weight, and intraperitoneal glucose tolerance test (IPGTT) in streptozotocin (STZ)-induced diabetic mice. Experiment design: following the induction of hyperglycemia using multiple low-dose STZ, treatment is administered: Control (Dulbecco's modified Eagle's medium, DMEM), conditioned medium from human exfoliated deciduous teeth (SHED-CM), exendin-4 (Ex-4), or bone marrow-derived mesenchymal stem cells (BM-CM) (A). Open arrows indicate STZ administration; closed arrows indicate days when the effectiveness of the treatment was evaluated. Random blood glucose levels (B) and body weight (C) were measured at 9:00. IPGTT was conducted on day 27 after $16 \mathrm{~h}$ of fasting, and blood glucose levels (D) and plasma insulin levels (E) were measured. Treatment is administered with control (DMEM), SHED-CM, or Ex-4 after the induction of hyperglycemia with STZ (glucose $\geq 20 \mathrm{mmol} / \mathrm{L}$ ) in high-dose STZ-induced diabetic mice. Random blood glucose levels (F) and body weight (G) were measured. Closed squares indicate control (DMEM); closed circles indicate SHED-CM; open squares indicate Ex-4; open circles indicate BM-CM; closed arrows indicate the period of treatment. The data are presented as a mean \pm SEM value. ${ }^{*} p<0.05$ versus control; $\uparrow p<0.05$ versus $E x-4 ; \S p<0.05$ versus $B M-C M$. 
measured using the CytoTox-96 cytosolic lactate dehydrogenase (LDH) assay (Promega UK, Ltd, Southampton, UK), according to the manufacturer's instructions. Total LDH release was calculated by incubating untreated cells with $9 \%$ Triton $\mathrm{X}-100$ at $37^{\circ} \mathrm{C}$ for $45 \mathrm{~min}$ to induce maximal cell lysis. Values for treated cells were then expressed as the percentage of the total LDH release. Background $\mathrm{LDH}$ release induced by media alone was subtracted from the experimental values. Cell viability was measured using the MTT (3- $(4,5-$ dimethylthiazol-2-yl)-2, 5-diphenyltetrazolium bromide) cell proliferation assay (Roche Diagnostics, Ltd, Burgess Hill, UK) according to the manufacturer's instructions. Briefly, the cells were incubated for $4 \mathrm{~h}$ in the presence of the MTT reagent and lysed with lysis buffer. After an overnight incubation, absorbance was measured at $\mathrm{A} 550 \mathrm{~nm}$ to $\mathrm{A} 690 \mathrm{~nm}$.

\section{Glucose-stimulated insulin secretion}

MIN6 cells were preincubated with a $2.8 \mathrm{mmol} / \mathrm{L} \mathrm{KRB}$ buffer for $30 \mathrm{~min}$ and stimulated with $16.7 \mathrm{mmol} / \mathrm{L}$ glucose for $30 \mathrm{~min}$. Supernatant as release and acidethanol extraction used content were measured using the HTRF Insulin assay (CisBio). The amount of secreted insulin was normalized to cellular insulin content.

\section{Statistical analysis}

Experimental results are expressed as the mean \pm SE. Comparisons among quantitative variable groups were performed with analysis of variance followed by the Tukey post hoc test using GraphPad Prism software (V.6.03; GraphPad Software, Inc, San Diego, California, USA). A probability ( $p$ ) value of $<0.05$ was considered statistically significant.

\section{RESULTS}

\section{SHED-CM treatment ameliorated glucose intolerance in STZ mice}

The goal of this study was to evaluate the therapeutic effects of the factors released from SHED on a mouse STZ-induced diabetic model. CM from SHED and BM were collected $48 \mathrm{~h}$ after culture in serum-free DMEM. There were no significant differences in the cellular survival of cells following incubation in serum-free media (see online supplementary figure S1). When evaluating the effect of SHED-CM treatment, serumfree DMEM served as the control. SHED-CM treatment was also compared with treatment with Ex-4 and BM-CM. Insulin levels in SHED-CM and BM-CM were undetectable, in spite of the high concentration $(421.5 \mathrm{ng} / \mathrm{mL})$ in DMEM supplemented with $10 \%$ fetal bovine serum (FBS), indicating that SHED-CM contained little substance from FBS (data not shown). The design of the experiment using MLDS mice is shown in figure 1A. From the 12th day after initiation of treatment, random plasma glucose levels in the SHED-CM
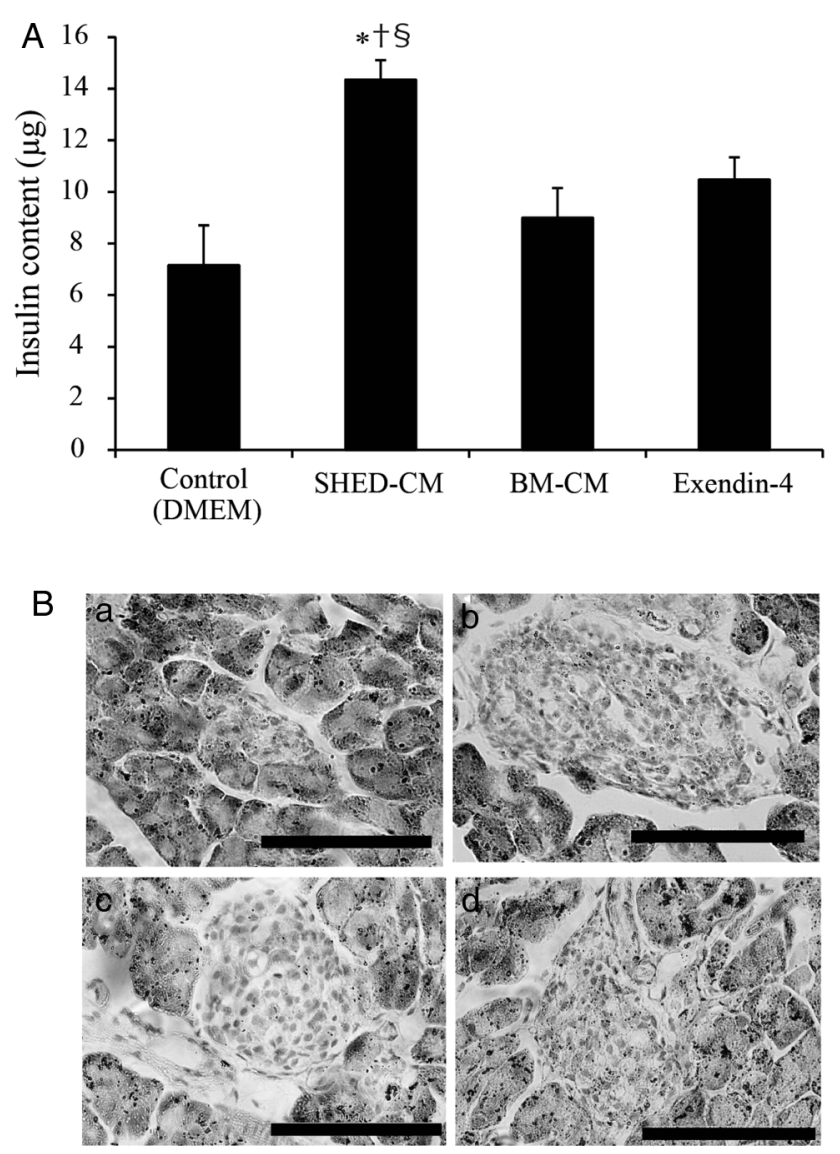

Figure 2 Insulin content of isolated pancreas and islet morphology. The insulin content of isolated pancreas was measured 3 weeks after the end of treatment (day 37) in multiple low-dose streptozotocin-induced diabetic mice (MLDS mice) (A). Pancreatic islets of the isolated pancreases were stained with H\&E on day 37 in MLDS mice (B). (a) Control (Dulbecco's modified Eagle's medium, DMEM); (b) conditioned medium from human exfoliated deciduous teeth (SHED-CM); (c) exendin-4 (Ex-4); and (d) bone marrow-derived mesenchymal stem cells (BM-CM). The data are presented as a mean \pm SEM value. Scale bars, $100 \mu \mathrm{m} .{ }^{*} \mathrm{p}<0.05$ versus control; $\uparrow p<0.05$ versus Ex-4; $\$ p<0.05$ versus BM-CM.

treatment group were the lowest among all of the treatment groups (figure 1B). There was no significant change in BW in any of the groups (figure 1C). The intraperitoneal glucose tolerance test (IPGTT) results showed that SHED-CM significantly reduced plasma glucose levels as measured on day 27 (the 13th day after the end of the treatment; figure 1D). SHED-CM also significantly increased plasma insulin levels in IPGTT on day 27 (figure 1E). Although the Ex-4 and BM-CM treatment groups showed reduced plasma glucose levels and increased plasma insulin levels in comparison with the control (DMEM) treatment group, SHED-CM was the most effective of the three treatments. To confirm that these effects were not due to an anti-STZ effect of the treatments, we explored the effects of these treatments in HD mice that were rendered hyperglycemic (random plasma glucose level $\geq 20 \mathrm{mmol} / \mathrm{L}$ ) with high-dose STZ injections before 
Figure 3 Immunohistochemical analysis. The pancreas was isolated 3 weeks after the end of treatment (day 37 ) in multiple low-dose streptozotocin-induced diabetic mice. Sections were immunostained for glucagon (red) and insulin (green) (A). (a) Control (Dulbecco's modified Eagle's medium, DMEM); (b) conditioned medium from human exfoliated deciduous teeth (SHED-CM); (c) exendin-4 (Ex-4); and (d) bone marrow-derived mesenchymal stem cells (BM-CM). Morphometric analysis of insulin-positive ( $\beta$-cell) areas and glucagon-positive ( $\alpha$-cell) areas was conducted $(B)$. The $\alpha$-cell $/ \beta$-cell area ratios are shown as a ratio of glucagon-positive to insulin-positive areas (C). The data are presented as a mean \pm SEM value. Scale bars, $100 \mu \mathrm{m}$. ${ }^{*} \mathrm{p}<0.05$ versus control; $\uparrow \mathrm{p}<0.05$ versus $\mathrm{Ex}-4 ; \S \mathrm{p}<0.05$ versus BM-CM.
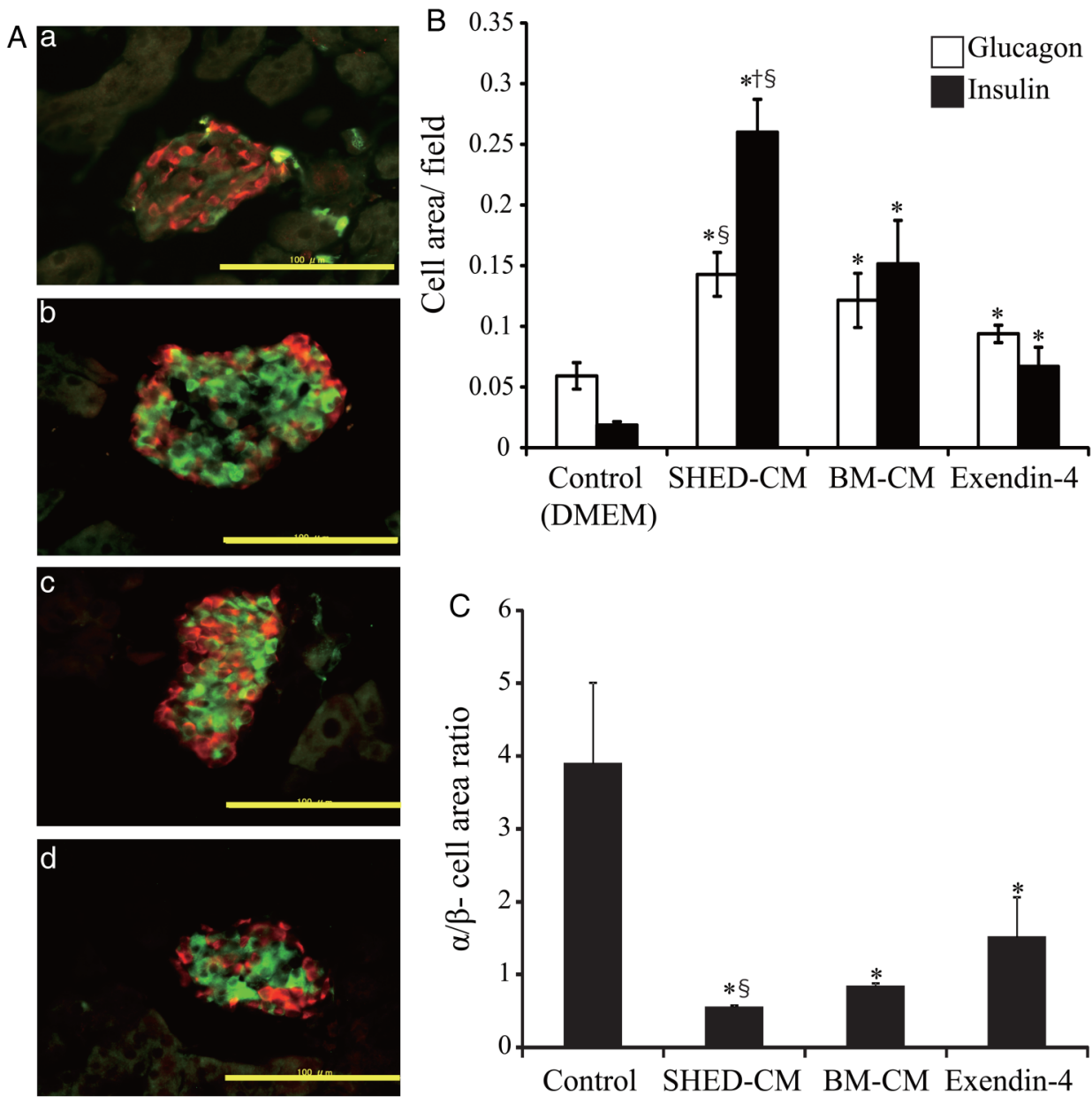

treatment. In the SHED-CM treatment group, random plasma glucose levels were significantly reduced (figure 1F) and BW loss was significantly suppressed compared with those in the control (DMEM) and Ex-4 groups (figure $1 \mathrm{G}$ ).

\section{Increase in pancreatic insulin content and insulin-positive cells in the SHED-CMI treatment group}

The in vivo data suggest that amelioration of insulin secretion possibly contributed to the improvement in glycemic regulation; thus, we further investigated the state of insulin preservation in pancreatic $\beta$-cells in MLDS mice. The insulin content of the isolated pancreas from mice in the SHED-CM treatment group was significantly higher than that in the other treatment groups (the insulin content for DMEM, SHED-CM, Ex-4, and BM-CM treatment groups was $7.16 \pm 1.53,14.3 \pm 0.74$, $10.4 \pm 0.86$, and $9.00 \pm 0.86 \mu \mathrm{g}$, respectively; figure $2 \mathrm{~A}) . \mathrm{HE}$ staining showed that SHED-CM treatment preserved the islet mass and maintained the normal round shape compared with the other treatment groups (figure 2B).

Immunofluorescent staining showed that the proportion of insulin-positive cells ( $\beta$-cells) increased significantly more in the SHED-CM treatment group than in other treatment groups (figure $3 \mathrm{~A}, \mathrm{~B}$ ). The ratio of $\alpha$-cell area to $\beta$-cell area, which is usually less than 1 in wild-type mice, was significantly less in the SHED-CM treatment group (figure 3C). Significant differences between the SHED-CM and BM-CM treatment groups were detected for all three parameters ( $\beta$-cell and $\alpha$-cell areas, and the ratio of $\alpha$-cell area to $\beta$-cell area). In the SHED-CM treatment group, the ratio of cells positive for Ki67 (a proliferation marker) to cells positive for insulin was significantly higher than that in the other treatment groups (percentages for DMEM, SHED-CM, Ex-4, and BM-CM treatment groups were $0.88 \pm 0.30,2.26 \pm 0.51,1.0 \pm 0.52$, and 0.58 \pm 0.16 , respectively; figure $4 \mathrm{~A}, \mathrm{~B})$. Neogenesis of pancreatic $\beta$-cells from pancreatic duct epithelial cells ${ }^{19}$ or precursor cells in islets $^{20}$ is reportedly implicated in the regeneration of pancreatic $\beta$-cells, ${ }^{19}$ although no pancreatic duct epithelial cell was co-stained by both anticytokeratin19 antibody and anti-insulin antibody in this study (data not shown). These data indicate that SHED-CM treatment increased the $\beta$-cell area through the proliferation of pancreatic $\beta$-cells.

\section{Direct effects of SHED-CM on pancreatic $\beta$-cell function and survival}

We conducted an in vitro study using MIN6 cells to clarify whether SHED-CM directly improved the viability and function of pancreatic $\beta$-cells. Nuclear staining showed that, in the control (DMEM) treatment group, 
Figure 4 Pancreatic $\beta$-cell proliferation. Sections were immunostained for Ki67 (red), insulin (green), and DAPI (blue) (A). The arrows point at Ki67+ cells. Scale bars, $100 \mu \mathrm{m}$. Mean \pm SEM percentage of Ki67+ cells/ insulin+ and DAPI+ cells (B). Scale bars, $100 \mu \mathrm{m} .{ }^{*} p<0.05$ versus control; $\uparrow p<0.05$ vs Ex-4; $\S p<0.05$ vs BM-CM. BM-CM, bone marrow-derived mesenchymal stem cells; DMEM, Dulbecco's modified Eagle's medium; Ex-4, exendin-4; SHED-CM, conditioned medium from human exfoliated deciduous teeth.
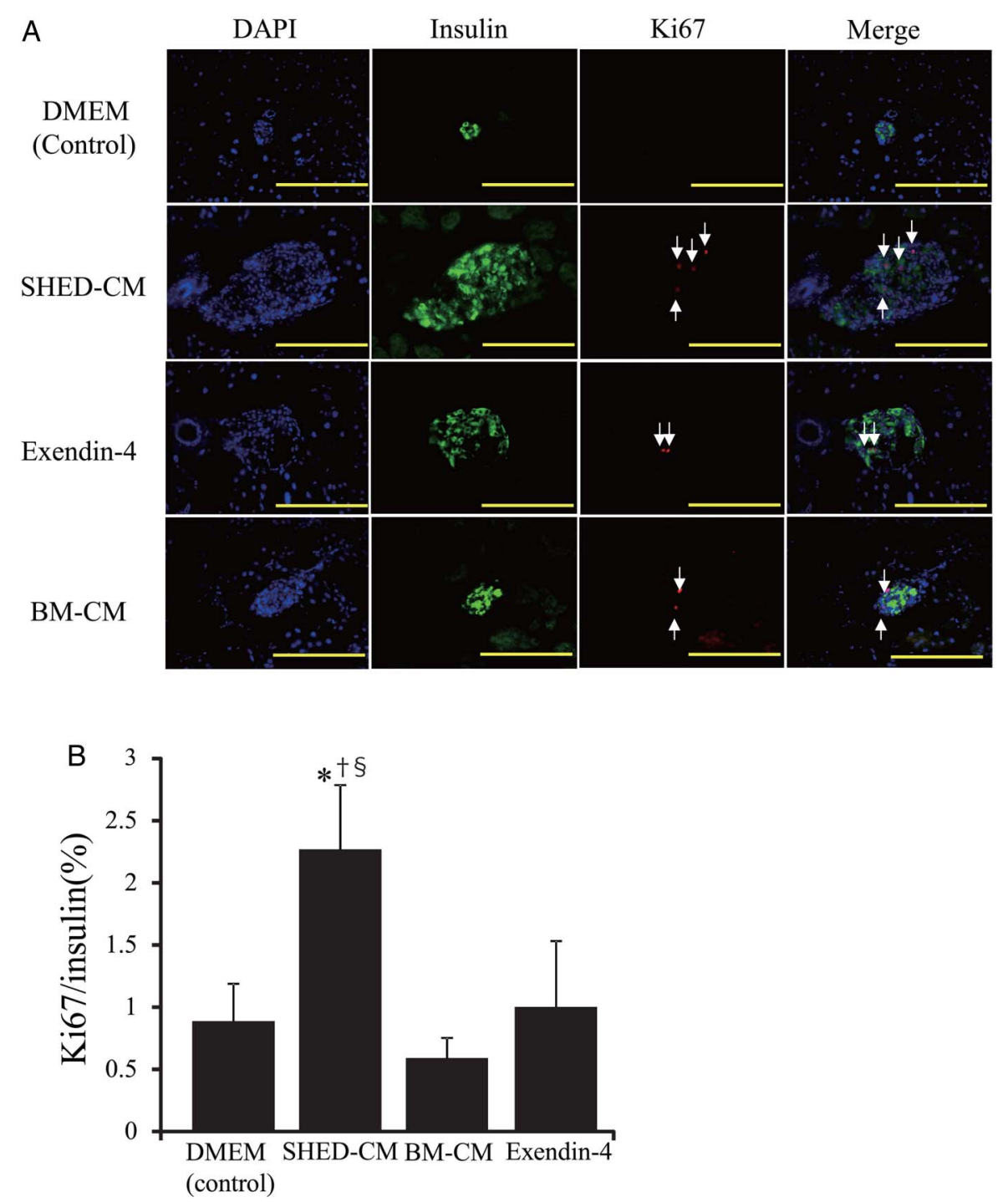

the percentage of swollen cells (early stage of necrosis cells) increased in an STZ concentration-dependent manner. Nevertheless, this was significantly suppressed in the SHED-CM treatment group (figure 5A). It is notable that SHED-CM treatment was more effective than either Ex-4 or BM-CM treatment. The results of the LDH assay showed that SHED-CM treatment significantly suppressed $\mathrm{LDH}$ release parallel to the numbers of cells with a swollen nucleus (figure $5 \mathrm{~B}$ ). These data indicated that SHED-CM treatment directly suppressed pancreatic $\beta$-cell death. Meanwhile, the results of the MTT assay showed that SHED-CM treatment significantly increased cell viability, as compared with the other treatments (figure 5C). Combined, these data suggested that SHED-CM treatment directly protected pancreatic $\beta$-cells from STZ-induced cell damage.

Next, we examined the effect of SHED-CM on the function of pancreatic $\beta$-cells. Glucose-stimulated insulin secretion (GSIS) in the control (DMEM) group was decreased in an STZ concentration level-dependent manner. Insulin secretion under high-glucose conditions was significantly increased in the SHED-CM treatment group compared with that in the other treatment groups (figure 5D). In addition, the results of the MTT assay (see online supplementary figure S2A) and GSIS (see online supplementary figure 2B) showed that SHED-CM was more effective than DMEM with $10 \%$ FBS, which contains many growth factors, indicating that SHED-CM has the original protective effect on pancreatic $\beta$-cells, even if a negligible amount of FBS may have contaminated SHED-CM. Taken together, these data strongly suggest that SHED-CM treatment directly increased the viability and function of pancreatic $\beta$-cells.

\section{DISCUSSION}

We experienced a previously unreported phenomenon. SHED-CM treatment regenerated pancreatic $\beta$-cells in STZ-induced diabetic mice, in which pancreatic $\beta$-cells were almost completely destroyed. This is the first report of factors secreted by stem cells that ameliorate the failure of pancreatic $\beta$-cells in vivo. Moreover, our data demonstrate that SHED-CM treatment directly improved both the survival and function of pancreatic $\beta$-cells. Our 

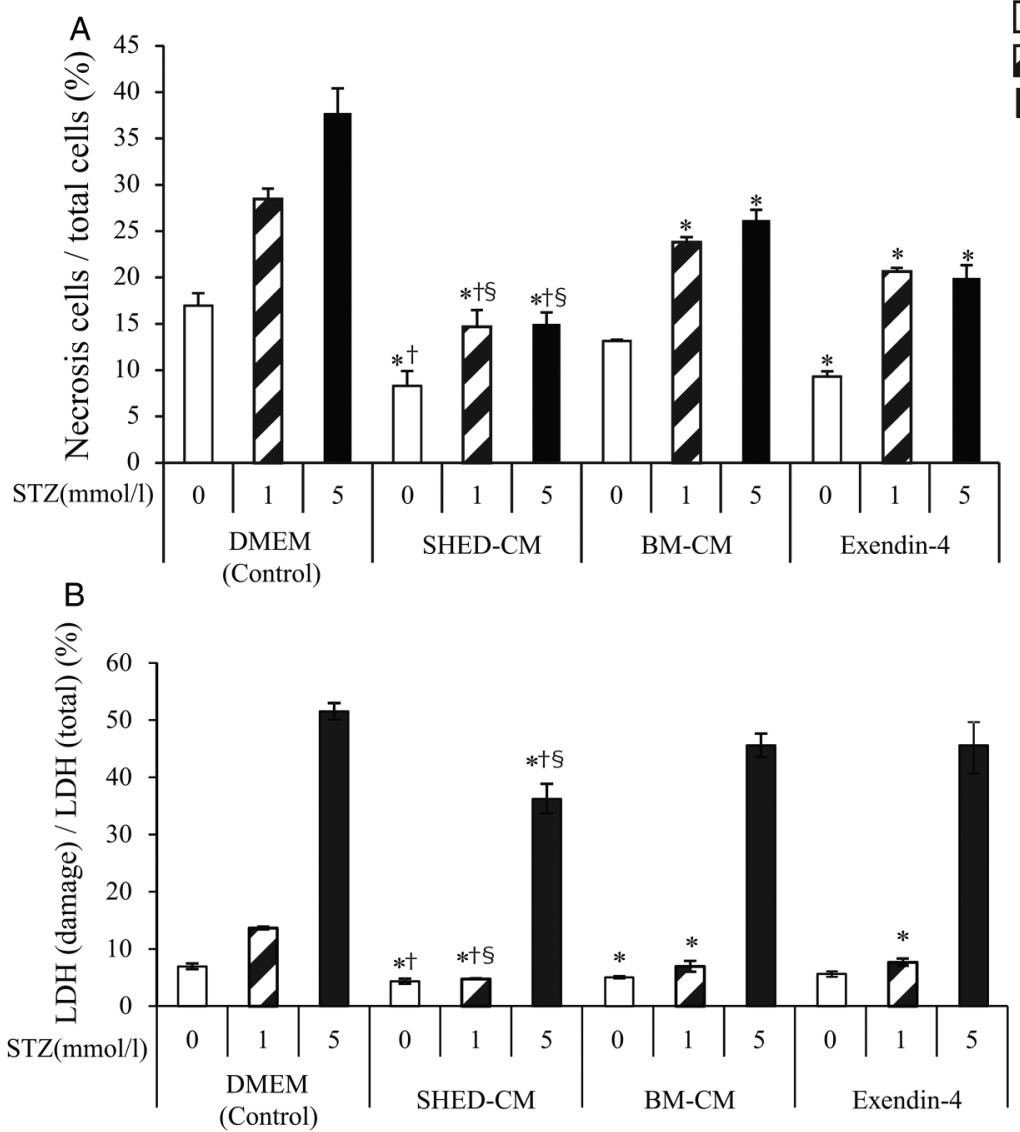

$\mathrm{STZ} 0 \mathrm{mmol} / \mathrm{l}$

STZ $0 \mathrm{mmol} / 1$

$\mathrm{STZ} 1 \mathrm{mmol} / 1$

STZ $5 \mathrm{mmol} / 1$
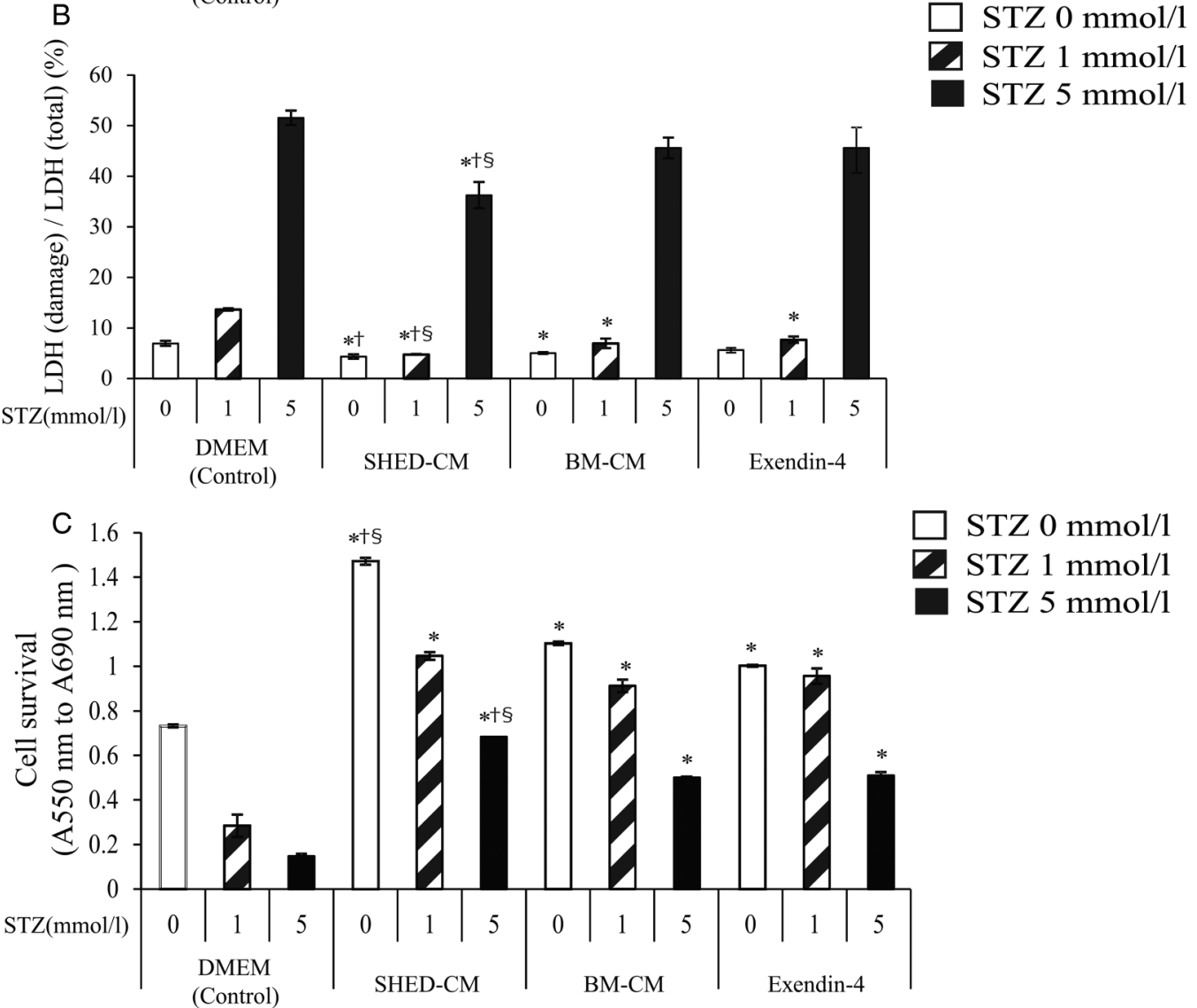

$\mathrm{STZ} 0 \mathrm{mmol} / 1$

$\mathrm{STZ} 1 \mathrm{mmol} / 1$

$\mathrm{STZ} 5 \mathrm{mmol} / 1$

Figure 5 Viability and function of mouse pancreatic cell line. MIN6 cells were cultured to reach $80 \%$ confluence, then incubated with different concentrations of streptozotocin (STZ; 0,1 , or $5 \mathrm{mmol} / \mathrm{L}$ ) with conditioned medium from human exfoliated deciduous teeth (SHED-CM), Dulbecco's modified Eagle's medium (DMEM), exendin-4 (Ex-4; $10 \mathrm{nmol} / \mathrm{L})$, or bone marrow-derived mesenchymal stem cells (BM-CM) for $6 \mathrm{~h}$. Cell damage were examined by nuclear DAPI staining $(A)$ and using the lactate dehydrogenase (LDH) assay (B). The ratio of the total cells to cells with a swollen nucleus was analyzed using the BZ-9000 (A). The results of the LDH assay are expressed as a percentage of LDH release (B). The MTT (3-(4, 5-dimethylthiazol-2-yl)-2, 5-diphenyltetrazolium bromide) assay was conducted to examine cell survival (C). The MIN6 cells were preincubated with $2.8 \mathrm{mmol} / / \mathrm{KRB}$ buffer for $30 \mathrm{~min}$, then stimulated with $2.8 \mathrm{mmol} / \mathrm{L}$ (low glucose) or $16.7 \mathrm{mmol} / \mathrm{L}$ (high glucose) for $30 \mathrm{~min}$ for glucose-stimulated insulin secretion (D). The data are presented as a mean $\pm S E M$ value. ${ }^{*} p<0.05$ versus control; $\dagger p<0.05$ versus Ex-4; $\S p<0.05$ versus BM-CM.

data suggest that factors secreted by stem cells (paracrine signals) are possible causes of this phenomenon. A very recent study reported that BM transplantation and BM-CM injection equally improved insulin resistance by inhibition of hepatocytic inflammation in mice fed with a high-fat diet, although the antihyperglycemic actions of these treatments were negligible. ${ }^{21}$ Cytosolic extract from regenerating pancreas ${ }^{22}$ and pancreatic $\beta$-cell culture supernatant ${ }^{20}$ were also reported to improve glycemic control in mice administered STZ. 

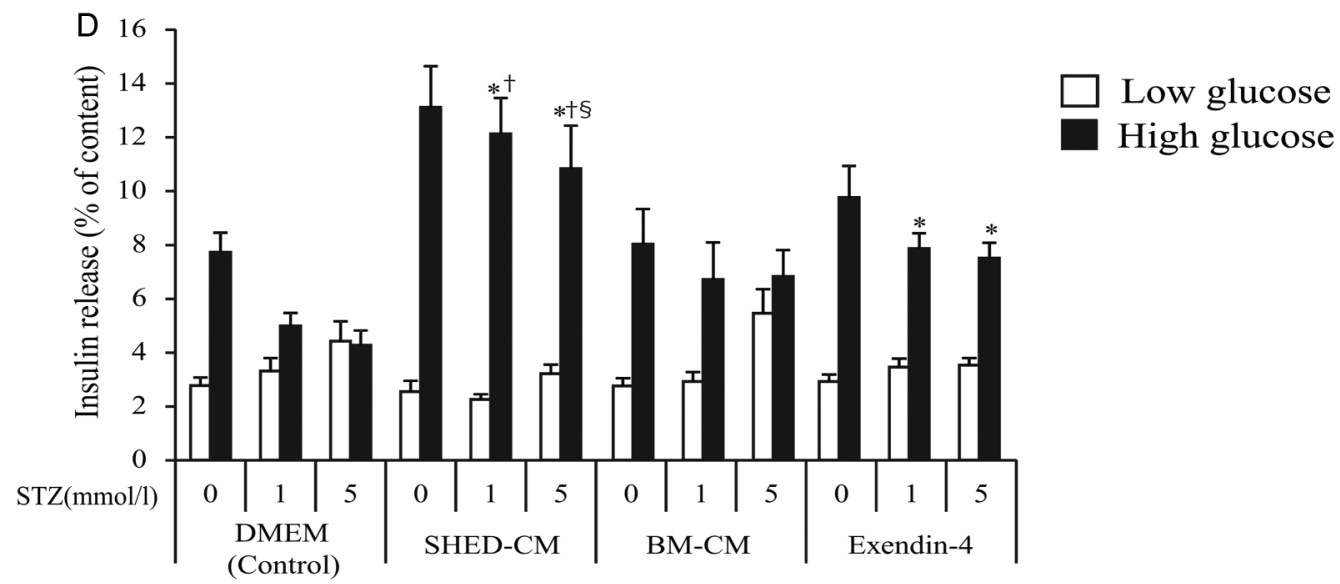

High glucose

Figure 5 Continued

These reports support the possibility that secreted factors may play important roles in reducing hyperglycemia, as found in the present study.

Stem cell-CM reportedly contains many different growth factors and anti-inflammatory chemokines. ${ }^{23}$ Bai et $a l^{24}$ reported that hepatocyte growth factor (HGF) secreted by BM can recover neurological function in multiple sclerosis model rats by promoting remyelination. ${ }^{24}$ Insulin-like growth factor (IGF)-1 secreted by BM improves lipopolysaccharide-induced mouse acute lung injury. ${ }^{25}$ Vascular endothelial growth factor (VEGF) secreted by dental pulp stem cells promotes the migration of endothelial cells (human mammary epithelial cells (HMEC)-1), which is attenuated by inactivation of phosphoinositide 3-kinase (PI3K). ${ }^{23}$ Previous studies have revealed that VEGF-A plays an important role in the proliferation and development of pancreatic $\beta$-cells, ${ }^{26}$ and the activation of PI3K suppresses cell death, promotes proliferation, and activates the function of pancreatic $\beta$-cells. ${ }^{27}$ Our preliminary examination of fractionated SHED-CM according to molecular weight showed that compared with DMEM, a fraction of more than $100 \mathrm{kDa}$ improved viability of STZ-treated MIN6 cells (see online supplementary figure S3A). Meanwhile, very recent studies reported that exosomes from deciduous dental pulp stem cells suppressed carrageenan-induced acute inflammation in mice, ${ }^{28}$ and exosomes from human umbilical cord MSCs improved acute myocardial ischemic injury in rats. ${ }^{29}$ The MTT assay results showed that compared with SHED-CM, SHED-CM without exosomes, which were eliminated from SHED-CM by centrifugation at $200000 \times \mathrm{g}$ for $110 \mathrm{~min}$, apparently reduced cell viability (see online supplementary figure 3B). Taken together, these data suggest that both soluble factors and exosome secretion by SHED contribute to the growth and function of pancreatic $\beta$-cells, although the exact mechanisms of these antidiabetic effects by SHED-CM need to be further elucidated. SHED-CM treatment was more effective than BM-CM treatment both in in vivo and in vitro studies. Therefore, a comparison of factors secreted by SHED and BM could lead to the identification of previously unknown molecules with antidiabetic effects.

In this study, although the large injected amount $(2 \mathrm{~mL} /$ day $)$ may have caused volume overload and affected physical function, none of the treated mice died or presented with hematuria or uric protein. Besides, SHED-CM showed the greatest extent of amelioration of glucose intolerance compared with that with an injection of the same volume of either DMEM or BM-CM, indicating that the secreted SHED, but not the large injected amount, affected the improvement in glucose intolerance. Moreover, SHED-CM without the unnecessary fractionation of less than $100 \mathrm{kDa}$ may be preferable to minimize the risk of unknown side effects in future studies.

The profound effect of SHED-CM treatment on pancreatic $\beta$-cells could be based on the characteristics of these stem cells, which differed from those used in all the other treatments. Pancreatic $\beta$-cells are derived from the endoderm, but neuronal cells and pancreatic $\beta$-cells have many similar characteristics, including the expression of common transcription factors, such as Pdx-1 and Ngn3, as well as cellular functions, such as secretion. ${ }^{30}$ SHED, which are dental pulp stem cells from exfoliated deciduous teeth, are derived from the neural crest and express Nestin, Dcx, and glial fibrillary acidic protein (GFAP) as neural crest stem cell markers, and A2B5 and CNPase as oligodendrocyte progenitor cell markers. ${ }^{9} 12$ It is well known that SHED cells are easily differentiated into neural tissue, ${ }^{9}$ and dental pulp stem cells have been reported to differentiate into pancreatic $\beta$-like cells, ${ }^{31}$ suggesting that SHED-CM contains commonly secreted factors influencing the mechanisms of differentiation, function, and secretion between neural cells and pancreatic $\beta$-cells. This may be why the SHED-CM treatment was more effective at prompting growth and insulin secretion in pancreatic $\beta$-cells than BM-CM treatment.

In conclusion, a strong proliferative effect, as well as promotion of insulin secretion, was observed in pancreatic $\beta$-cells as a result of SHED-CM treatment. SHED-CM treatment induced insulin secretion in relation to 
glucose concentration. This treatment had much fewer side effects than cell transplantation and requires no immunosuppressive agents, indicating that SHED-CM treatment may be a safer, less invasive method of treating diabetes. Furthermore, the antidiabetic effects of SHED-CM treatment were much more pronounced than those of treatments with Ex-4 and BM-CM. SHED-CM treatment may have potential as a new, practical strategy for diabetic treatment. Nonetheless, future studies should be performed to investigate the mechanism of action and effectiveness of SHED-CM treatment for clinical applications in the treatment of diabetes.

\section{Author affiliations}

${ }^{1}$ Department of Endocrinology and Diabetes, Nagoya University Graduate School of Medicine, Nagoya, Aichi, Japan

${ }^{2}$ Department of Oral and Maxillofacial Surgery, Nagoya University Graduate School of Medicine, Nagoya, Aichi, Japan

${ }^{3}$ Department of Metabolic Medicine, Nagoya University Graduate School of Medicine, Nagoya, Aichi, Japan

Acknowledgements The authors would like to thank Dr Junichi Miyazaki of the Osaka University Graduate School of Medicine (Osaka, Japan) for the kind gift of MIN6 cells, and Michiko Yamada of the Nagoya University Graduate School of Medicine (Nagoya, Japan) for her technical assistance.

Contributors TI-A, ST, AY, EU, YS, HH, HA, MU, and YO conceived the experimental design and interpreted the data. TI-A and ST wrote the manuscript. TI-A, ST, YS, KI, HO, AI, MI, KH, YN, YY, and RM acquired the data. TI-A, KI, EU, and $\mathrm{YH}$ analyzed the data. All authors reviewed/edited the manuscript and gave final approval of the version to be published. ST is the guarantor of this work and, as such, had full access to all of the data presented in this study and takes responsibility for the integrity of the data and the accuracy of data analysis.

Competing interests None declared.

Ethics approval The study protocol was approved by the Ethics Committee of Nagoya University.

Provenance and peer review Not commissioned; externally peer reviewed.

Data sharing statement No additional data are available.

Open Access This is an Open Access article distributed in accordance with the Creative Commons Attribution Non Commercial (CC BY-NC 4.0) license, which permits others to distribute, remix, adapt, build upon this work noncommercially, and license their derivative works on different terms, provided the original work is properly cited and the use is non-commercial. See: http:// creativecommons.org/licenses/by-nc/4.0/

\section{REFERENCES}

1. Mokdad AH, Bowman BA, Ford ES, et al. The continuing epidemics of obesity and diabetes in the United States. JAMA 2001:286:1195-200.

2. Rhodes CJ. Type 2 diabetes-a matter of beta-cell life and death? Science 2005;307:380-4

3. Shapiro AM, Lakey JR, Ryan EA, et al. Islet transplantation in seven patients with type 1 diabetes mellitus using a glucocorticoid-free immunosuppressive regimen. N Engl J Med 2000;343:230-8.

4. Urban VS, Kiss J, Kovacs J, et al. Mesenchymal stem cells cooperate with bone marrow cells in therapy of diabetes. Stem Cells 2008;26:244-53.

5. Cantinieaux D, Quertainmont R, Blacher S, et al. Conditioned medium from bone marrow-derived mesenchymal stem cells improves recovery after spinal cord injury in rats: an original strategy to avoid cell transplantation. PLOS ONE 2013;8:e69515.

6. Yamagata M, Yamamoto A, Kako E, et al. Human dental pulp-derived stem cells protect against hypoxic-ischemic brain injury in neonatal mice. Stroke 2013;44:551-4.
7. Ranganath $\mathrm{SH}$, Levy O, Inamdar MS, et al. Harnessing the mesenchymal stem cell secretome for the treatment of cardiovascular disease. Cell Stem Cell 2012;10:244-58.

8. Lee T. Stem cell therapy independent of stemness. World J Stem Cells 2012;4:120-4.

9. Miura M, Gronthos S, Zhao M, et al. SHED: stem cells from human exfoliated deciduous teeth. Proc Natl Acad Sci USA 2003;100:5807-12.

10. Gronthos S, Mankani M, Brahim J, et al. Postnatal human dental pulp stem cells (DPSCs) in vitro and in vivo. Proc Natl Acad Sci USA 2000;97:13625-30.

11. Huang GT, Gronthos S, Shi S. Mesenchymal stem cells derived from dental tissues vs. those from other sources: their biology and role in regenerative medicine. J Dent Res 2009;88:792-806.

12. Sakai K, Yamamoto A, Matsubara K, et al. Human dental pulp-derived stem cells promote locomotor recovery after complete transection of the rat spinal cord by multiple neuro-regenerative mechanisms. J Clin Invest 2012;122:80-90.

13. Kanafi MM, Rajeshwari YB, Gupta S, et al. Transplantation of islet-like cell clusters derived from human dental pulp stem cells restores normoglycemia in diabetic mice. Cytotherapy 2013;15:1228-36.

14. Tsunekawa S, Demozay D, Briaud I, et al. FoxO feedback control of basal IRS-2 expression in pancreatic $\beta$-cells is distinct from that in hepatocytes. Diabetes 2011;60:2883-91.

15. Tsunekawa S, Yamamoto N, Tsukamoto K, et al. Protection of pancreatic beta-cells by exendin-4 may involve the reduction of endoplasmic reticulum stress; in vivo and in vitro studies. J Endocrinol 2007;193:65-74.

16. Iwasaki M, Minami K, Shibasaki T, et al. Establishment of new clonal pancreatic beta-cell lines (MIN6-K) useful for study of incretin/cyclic adenosine monophosphate signaling. J Diabetes Investig 2010;1:137-42

17. Lenzen S. The mechanisms of alloxan- and streptozotocin-induced diabetes. Diabetologia 2008;51:216-26.

18. Golstein P, Kroemer G. Cell death by necrosis: towards a molecular definition. Trends Biochem Sci 2007;32:37-43.

19. Pagliuca FW, Melton DA. How to make a functional beta-cell. Development 2013;140:2472-83.

20. Kanitkar M, Bhonde R. Existence of islet regenerating factors within the pancreas. Rev Diabet Stud 2004;1:185-92.

21. Nagaishi K, Ataka K, Echizen E, et al. Mesenchymal stem cell therapy ameliorates diabetic hepatocyte damage in mice by inhibiting infiltration of bone marrow-derived cells. Hepatology 2014;59:1816-29.

22. Hardikar AA, Bhonde RR. Modulating experimental diabetes by treatment with cytosolic extract from the regenerating pancreas. Diabetes Res Clin Pract 1999;46:203-11.

23. Bronckaers $A$, Hilkens $P$, Fanton $Y$, et al. Angiogenic properties of human dental pulp stem cells. PLoS ONE 2013;8:e71104.

24. Bai L, Lennon DP, Caplan Al, et al. Hepatocyte growth factor mediates mesenchymal stem cell-induced recovery in multiple sclerosis models. Nat Neurosci 2012;15:862-70.

25. Ionescu L, Byrne RN, van Haaften T, et al. Stem cell conditioned medium improves acute lung injury in mice: in vivo evidence for stem cell paracrine action. Am J Physiol Lung Cell Mol Physiol 2012;303:L967-77.

26. Brissova M, Aamodt $\mathrm{K}$, Brahmachary $\mathrm{P}$, et al. Islet microenvironment, modulated by vascular endothelial growth factor-A signaling, promotes beta cell regeneration. Cell Metab 2014;19:498-511.

27. Elghazi L, Rachdi L, Weiss AJ, et al. Regulation of beta-cell mass and function by the Akt/protein kinase B signalling pathway. Diabetes Obes Metab 2007;9(Suppl 2):147-57.

28. Pivoraite $U$, Jarmalaviciute $A$, Tunaitis $\mathrm{V}$, et al. Exosomes from human dental pulp stem cells suppress carrageenan-induced acute inflammation in mice. Inflammation 2015;38:1933-41.

29. Zhao $Y$, Sun $X$, Cao $W$, et al. Exosomes derived from human umbilical cord mesenchymal stem cells relieve acute myocardial ischemic injury. Stem Cells Int 2015;2015:761643.

30. Arntfield ME, van der Kooy D. $\beta$-Cell evolution: how the pancreas borrowed from the brain: the shared toolbox of genes expressed by neural and pancreatic endocrine cells may reflect their evolutionary relationship. Bioessays 2011;33:582-7.

31. Govindasamy V, Ronald VS, Abdullah AN, et al. Differentiation of dental pulp stem cells into islet-like aggregates. J Dent Res 2011;90:646-52. 